\title{
Dual RAS Therapy Not on Target, but Fully Alive
}

\author{
H.J. Lambers Heerspink $\quad$ D. de Zeeuw
}

Department of Clinical Pharmacology, University Medical Centre Groningen, University of Groningen, Groningen, The Netherlands

\section{Key Words}

Chronic kidney disease $\cdot$ Renin-angiotensin system •

Hyperkalemia

\begin{abstract}
Inhibitors of the renin-angiotensin system (RAS) form a cornerstone in the treatment of kidney disease. These drugs lower blood pressure and albuminuria, and afford renal protection. Dual therapy with an angiotensin-converting enzyme inhibitor and angiotensin receptor blocker have been shown to be more effective in reducing blood pressure and albuminuria than the single use of these agents. It was therefore expected that the combination of these drugs could delay the progression of renal disease. However, the ONTARGET renal analysis suggests that the use dual-agent RAS leads to increased renal risk. This led to vivacious discussions about the benefits and risks of dual-agent RAS in patients with nephropathy. We will review the ONTARGET trial design and interpretation, and offer implications for novel trials.
\end{abstract}

Copyright $\odot 2010$ S. Karger AG, Base
The results of the renal outcomes of the ONTARGET trial led to lively discussions among nephrologists as to whether dual-agent renin-angiotensin system (RAS) blockade is a safe and effective treatment strategy in patients with chronic kidney disease (CKD) [1-6]. In this review we contribute to these discussions offering a carefully described overview of the design of the ONTARGET trial and provide a novel perspective on the implications of the ONTARGET results for future trials.

Historically it was thought that the RAS consists of a number of circulating hormones mediating vasoconstriction, glomerular filtration pressure and aldosterone excretion. However, during recent years it has become increasingly apparent that the RAS has a broad diversity of (patho) physiological effects including modulation of inflammation and regulation of cell growth and differentiation [7, 8]. Since the kidney is both the endocrine organ and target organ of the RAS, the deleterious effects of angiotensin II, the primary mediator of the RAS, are profoundly apparent in the kidney. Indeed, continued activation of the RAS constricts renal arterioles leading to increased peripheral and renal resistance and triggers pro-inflammatory and pro-fibrotic processes in the kidney contributing to progressive renal function loss $[9,10]$. The importance of the RAS in renal disease has received further recognition by the proven clinical benefits of agents intervening in the

H.J. Lambers Heerspink, Department of Clinical Pharmacology University Medical Centre Groningen, University of Groningen Ant Deusing laan 1, NL-9713 AV Groningen (The Netherlands) Tel. +31 50363 2810, Fax +31503632812

E-Mail h.j.lambers.heerspink@med.umcg.nl 
RAS. The blood pressure-lowering effects of angiotensinconverting enzyme inhibitors (ACEI) and angiotensin receptor blockers (ARBs) are well documented and they have been demonstrated to improve clinical outcomes in both diabetic and non-diabetic renal disease [11-14].

Elevated levels of albuminuria can be detected in early stages of kidney disease and its presence is associated with progressive renal function loss. Besides the blood pressure-lowering effects of agents intervening in the RAS, they also appear to lower albuminuria. A systematic review of clinical trials with ACEIs and ARBs has shown that either agent reduces albuminuria by approximately $35 \%$ [15]. The reduction in albuminuria achieved with RAS blockade may be a critical step towards achieving renoprotection. In the early work of Rossing et al. [16] and Apperloo et al. [17] it was shown that the magnitude of albuminuria reduction during the first months of therapy with an ACEI in patients with diabetic and non-diabetic disease was linearly associated with the degree of estimated glomerular filtration rate loss decline during long-term follow-up. This observation was later on confirmed in individuals with diabetic nephropathy. Posthoc analysis of the IDNT and RENAAL trial showed that the degree of the initial albuminuria reduction by irbesartan or losartan, respectively, was the most important predictor for long-term renal protection and turned out to be independent of changes in blood pressure [18-20]. Similar observations were made in the IRMA-2 trial, evaluating the effect of the angiotensin-II antagonist irbesartan [21]. This trial in hypertensive type 2 diabetic individuals at early stages of kidney disease showed that albuminuria can be substantially lowered and that this is associated with a lower rate of progression of kidney disease. The renoprotective effects of irbesartan observed in this trial appeared to be independent of blood pressure, as assessed by 24 -hour ambulatory blood pressure measurement [22]. Interestingly, the relation between initial albuminuria reduction and long-term renoprotection is not only observed in individuals participating in a trial but also on a meta-trial level. A joint analysis of multiple randomized clinical trials investigating the effects of RAS inhibitors on renal disease progression illustrates that the larger the albuminuria reduction achieved during the first months of treatment within a trial, the higher the relative risk reduction for renal events in that trial during long-term follow-up (fig. 1).

The aforementioned studies indicate that lowering albuminuria with ACEI or ARB therapy is associated with renoprotection independent of blood pressure changes. However, although of interest, these studies are post-hoc analyses of clinical trials which were not specifically designed to demonstrate that targeting albuminuria result in long-term renoprotection. More direct evidence that treatment regimens pursuing maximal reduction of albuminuria afford renoprotection comes from a study in subjects with non-diabetic kidney disease. Hou et al. [12] demonstrated that titrating the dose of an ACEI or ARB to maximal albuminuria lowering did not further lower blood pressure but delayed the rate of progressive renal function decline. These data thus support treatment strategies focusing on lowering albuminuria to levels as low as possible to achieve maximal renoprotection.

Dual therapy with both an ACEI and an ARB has been shown to be more effective in reducing albuminuria and blood pressure than single therapy with either an ACEI or ARB. One of the earliest studies in which the albuminuria response to a combination of an ACEI and ARB (candesartan $16 \mathrm{mg}+$ lisinopril $20 \mathrm{mg}$ ) was compared versus single-agent therapy reported an additional 34\% (95\% CI: 3-55) reduction in albuminuria versus ARB therapy and an additional 18\% (95\% CI: -20 to 44 ) reduction versus ACEI therapy [23]. Since then, numerous studies have confirmed that combination therapy is more beneficial in terms of blood pressure and albuminuria reduction than single-agent RAS therapy. These studies have been systematically reviewed by Kunz et al. [15]. This systematic review illustrated an overall additional $25 \%$ reduction in albuminuria with combined ACEI and ARB therapy versus single-agent therapy. These data have led to an expectation that, when compared to singleagent RAS blockade, dual therapy will provide greater protection against major adverse renal outcomes. However, the results of studies on hard outcomes are scarce and those conducted to date are invalid or show unanticipated results.

The COOPERATE trial in patients with CKD and high levels of albuminuria reported that the combination of an ACEI and ARBs confers renal protection in terms of fewer patients reaching dialysis [24]. However, serious concerns about the validity of the COOPERATE trial have been raised which resulted in an institutional investigation into the COOPERATE trial $[25,26]$. The investigational committee reviewed all medical records of the patients participating in the COOPERATE trial and concluded that it was unable to prove the authenticity of the data' [27]. The conclusions of this investigation mean that the COOPERATE trial cannot be used anymore for clinical decision-making. 


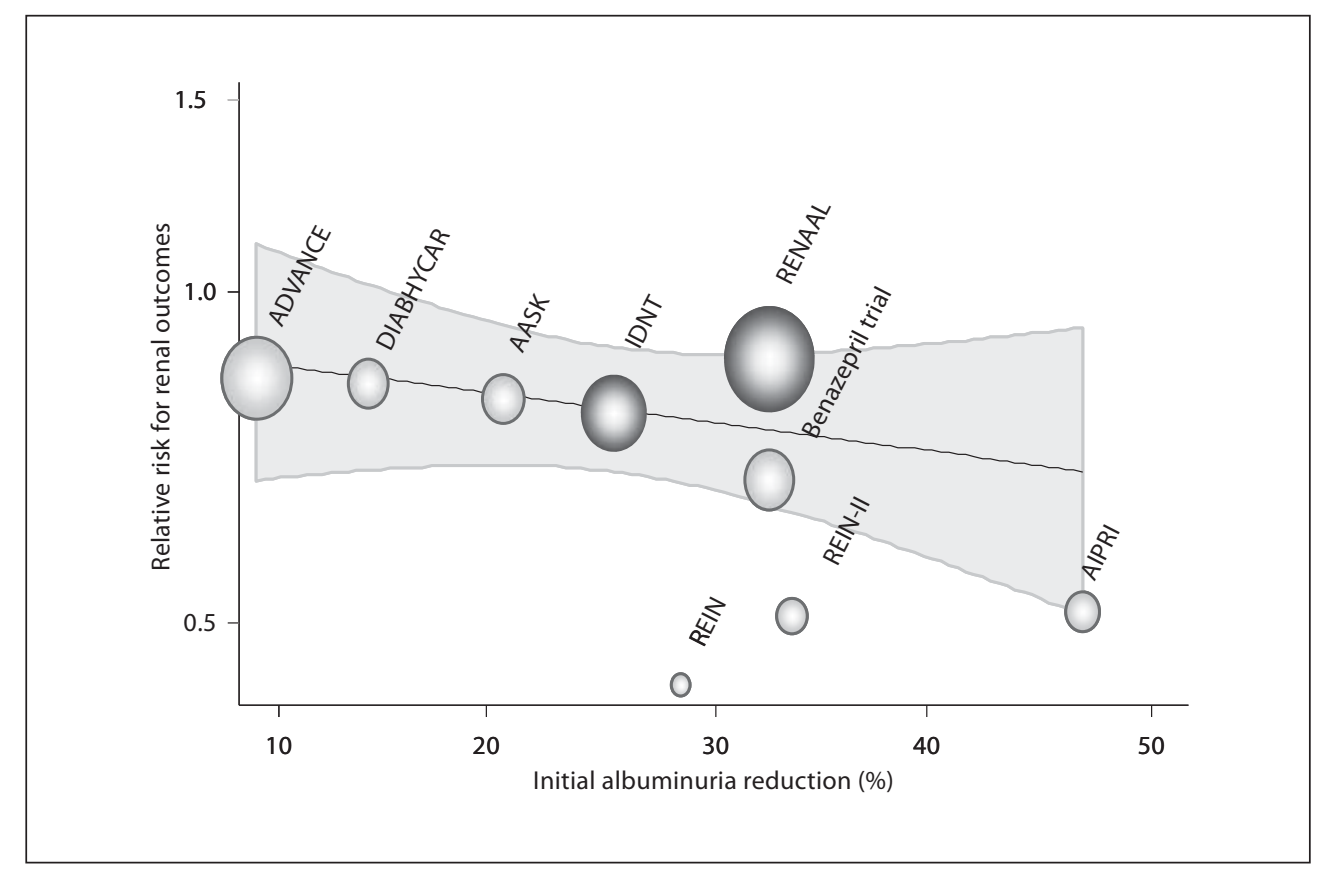

Fig. 1. Change in risk for renal outcomes and association with treatment induced reduction in albuminuria. Dark gray circles represent angiotensin receptor blocker trials, light gray circles ACEI trials. Gray area represents 95\% CI. The reduction in albuminuria during the first months of treatment and renal outcome was calculated relative to placebo except for the AASK trial in which it was calculated versus calcium channel blocker. Random effects meta-regression analysis with inverse variance weighting was conducted with STATA. Renal outcomes were defined as the composite of a doubling of serum creatinine or ESRD except for the AIPRI trial in which doubling of serum creatinine or chronic dialysis was recorded, the REIN trial in which only ESRD was recorded, and the ADVANCE trial in which the composite of macroalbuminuria, doubling of serum creatinine and ESRD was used. AASK = African American Study of Kidney Disease and Hypertension; ADVANCE = Action in Diabetes and Vascular Disease: preterAx and diamicroN-MR; AIPRI = AngiotensinConverting Enzyme Inhibition in Progressive Renal Insufficiency; DIABHYCAR = Diabetes, Hypertension, Cardiovascular Events and Ramipril; IDNT = Irbesartan Diabetic Nephropathy Trial; REIN = Ramipril Efficacy in Nephropathy, stratum I respectively II. RENAAL = Reduction of Endpoints in NIDDM with the Angiotensin II Antagonist Losartan.
Recently, the ONTARGET trial was published [28]. The ONTARGET trial included patients at risk for cardiovascular disease rather than kidney disease and randomly assigned these individuals to treatment with ramipril $10 \mathrm{mg} /$ day, telmisartan $80 \mathrm{mg} /$ day or the combination of both. As expected, systolic and diastolic blood pressure levels were 2.4 and $1.4 \mathrm{~mm} \mathrm{Hg}$ lower in the combination therapy group compared to ramipril 10 $\mathrm{mg} /$ day. Likewise, the increase in albuminuria level was also lower in the individuals assigned to combination therapy. However, despite the lower blood pressure and smaller increase in albuminuria, the combination of an ACEI and ARB did not afford cardiovascular protection in this population. Furthermore, a post-hoc analysis of this trial suggested that combination therapy increased the risk for renal disease [29]. After 56 months of followup the primary renal outcome had occurred in 1,233
(14.5\%), 1,147 (13.4\%) and 1,150 (13.5\%) individuals assigned to combination therapy, telmisartan $80 \mathrm{mg} / \mathrm{day}$, or ramipril $10 \mathrm{mg} /$ day, respectively. The results of the ONTARGET trial have led several authors to state that 'dual RAS therapy is dead' and that albuminuria is not a valid surrogate for renal protection [5]. However, such general and firm conclusions, based on a specific trial including a specific population, negate the value of all the single RAS inhibition therapy trials where the relation between the initial fall of albuminuria and long-term outcome has been demonstrated in different patient populations.

A thorough review of the ONTARGET trial is therefore warranted to put the results and subsequent communications into perspective. Furthermore, the renal outcome data of the ONTARGET trial have to be interpreted with some care, since the trial was clearly not set up a 
priori to look at renal endpoints. The validity of the results can therefore be questioned for a number of reasons:

- The trial counted all renal failure events, both acute and chronic, towards its renal endpoint. The majority of the adverse effects were observed for acute dialysis, which is potentially reversible, and is an outcome of less significance than long-term dialysis for end-stage renal disease (ESRD). No separate effect on the risk of chronic renal failure (beneficial or harmful) was demonstrated.

- Very few patients had high albuminuria levels at entry into the trial. Previous studies have shown that ACEIs and ARBs are mainly effective in slowing the progression of renal disease in patients with high albuminuria ( $\geq 500 \mathrm{mg} /$ day) [30]. A non-significant trend towards a beneficial effect of combination therapy was observed in the subgroup of patients with diabetic nephropathy and high albuminuria in $\mathrm{ON}$ TARGET, suggesting that combination therapy may be effective in those patients at highest risk for ESRD and cardiovascular events. In the ONTARGET study, the number of patients with albuminuria was too small to provide a clear result for this important group of patients.

- There were no clear guidelines for the management of hyperkalemia, hypotension or changes in kidney function. The effect of combination therapy in ONTARGET was accompanied by increases in serum potassium as well as with hypotensive periods, both of them adding to an increased renal risk [28]. Specific guidelines for the management of hyperkalemic episodes during the trial were lacking as well, which may have increased the risk for renal events.

- The ONTARGET trial was designed with an active run-in period meaning that all patients received active treatment during the 2 weeks prior to randomization to assess tolerability and treatment compliance. As well as increasing the risk of adverse outcomes for participants, it is possible that participants who are sensitive to RAS blockade, and are therefore most likely to benefit, were withdrawn from treatment during the run-in period due to elevations in serum creatinine, hyperkalemia, or hypotension. This may have had a substantial impact on the risk-benefit ratio observed in this trial.

A mostly unaddressed and unperceived topic in the interpretation of the ONTARGET trial and discussion about the validity of albuminuria being a surrogate for renal disease is that the same applies to high blood pressure. Blood pressure levels were lower in the combi- nation group than the single RAS therapy group. Nevertheless, both the renal and cardiovascular risk was higher in the combination group. Following the same reasoning as has been done for albuminuria, this would imply that blood pressure is not a valid surrogacy for renal and cardiovascular disease anymore, as increased blood pressure lowering using ACEi and ARB combination worsens cardiovascular and renal outcome according to the ONTARGET data. Of course, we are not going to send a message to stop using blood pressure as a surrogate on the basis of one trial, or one drug combination, as we also should not stop advocating the use of albuminuria as a potential valid surrogate on the basis of this trial.

How should we then interpret the ONTARGET trial and what lessons can we learn for the future? One of the most important lessons would be that we should evaluate and decide about trial results only for the patient group that was studied. Secondly, we need to realize that drugs or combinations of drugs targeting the same system, or increases in the doses of drugs may not automatically lead to better protection. In fact, drugs seldom have one effect. ACEIs and ARBs indeed have at least two effects which are both organ-protective: blood pressure lowering and albuminuria lowering. However, these drugs also have other effects among which increasing serum potassium is one that may actually increase cardiovascular (and even renal) risk. Studies in individuals with CKD have shown that the tolerability of dual RAS blockade is low due to an increased incidence in hyperkalemic episodes and symptomatic hypotension $[31,32]$. With regard to the occurrence of hypotensive episodes, the ACE inhibition in progressive renal disease collaboration indeed showed that a blood pressure goal of $<110 \mathrm{~mm} \mathrm{Hg}$ was associated with a higher risk for renal disease progression [33]. These adverse effects increase the risk for cardiovascular (and renal) outcomes and may offset the potential benefits of ACEI/ARB combination therapy. Thus, although the blood pressure- and albuminurialowering effects in ONTARGET may have been good, the increased incidence of hypotension and hyperkalemia (and maybe other things that were not measured) were apparently bad enough to make the combination treatment arm in the ONTARGET trial less effective than the single RAS comparator arms, in particular in a population in which albuminuria was not such an important risk factor.

As many patients with CKD still suffer from insufficient blood pressure and albuminuria control, they are consequently still at high risk for renal and cardiovascu- 
lar complications [34]. Novel treatment strategies are therefore still urgently required to lower this risk. Given the additional albuminuria-lowering effects of dualagent RAS therapy, it may still confer additional protection against hard outcomes. However, before such a strategy can be broadly applied in clinical practice, we should find new ways, and prove them to be effective, to maxi- mize the effects of combination therapy on the good surrogates and minimize the effects on the bad surrogates. We are thus patiently awaiting the results of several trials, VA NEPHRON-D [NCT 00494815], LIRICO [35], and HALT PKD [NCT00283686], assessing the balance of benefits and harms of dual-agent RAS blockade on renal and cardiovascular outcomes.

\section{References}

1 Cohen DL, Townsend RR: Is there added value to adding ARB to ACE inhibitors in the management of CKD? J Am Soc Nephrol 2009;20:1666-1668.

-2 Parving $\mathrm{HH}$, Brenner BM, McMurray JJ, de Zeeuw D, Haffner SM, Solomon SD, Chaturvedi N, Pfeffer MA: Dual renin-angiotensin system blockade and kidney disease. J Am Coll Cardiol 2009;54:278-280.

-3 Ruggenenti P, Remuzzi G. Proteinuria: Is the ONTARGET renal substudy actually off target? Nat Rev Nephrol 2009;5:436-437.

-4 Epstein M: Re-examining RAS-blocking treatment regimens for abrogating progression of chronic kidney disease. Nat Clin Pract Nephrol 2009;5:12-13.

$\checkmark 5$ Messerli FH: The sudden demise of dual renin-angiotensin system blockade or the soft science of the surrogate end point. J Am Coll Cardiol 2009;53:468-470.

-6 Berns JS: Is angiotensin-converting enzyme inhibitor and angiotensin receptor blocker combination therapy better than monotherapy and safe in patients with CKD? Am J Kidney Dis 2009;53:192-196.

7 Ruiz-Ortega M, Lorenzo O, Suzuki Y, Ruperez M, Egido J: Proinflammatory actions of angiotensins. Curr Opin Nephrol Hypertens 2001;10:321-329.

-8 Suzuki Y, Ruiz-Ortega M, Gomez-Guerrero C, Tomino Y, Egido J: Angiotensin II, the immune system and renal diseases: another road for RAS? Nephrol Dial Transplant 2003; 18:1423-1426.

-9 Remuzzi G, Bertani T: Pathophysiology of progressive nephropathies. N Engl J Med 1998;339:1448-1456.

10 Yoshioka T, Rennke HG, Salant DJ, Deen WM, Ichikawa I: Role of abnormally high transmural pressure in the permselectivity defect of glomerular capillary wall: a study in early passive Heymann nephritis. Circ Res 1987;61:531-538.

11 Ruggenenti P, Perna A, Gherardi G, Garini G, Zoccali C, Salvadori M, Scolari F, Schena FP, Remuzzi G: Renoprotective properties of ACE-inhibition in non-diabetic nephropathies with non-nephrotic proteinuria. Lancet 1999;354:359-364.

12 Hou FF, Zhang X, Zhang GH, Xie D, Chen PY, Zhang WR, Jiang JP, Liang M, Wang GB, Liu ZR, Geng RW: Efficacy and safety of benazepril for advanced chronic renal insufficiency. N Engl J Med 2006;354:131-140.

-13 Brenner BM, Cooper ME, de Zeeuw D, Keane WF, Mitch WE, Parving HH, Remuzzi G, Snapinn SM, Zhang Z, Shahinfar S: Effects of losartan on renal and cardiovascular outcomes in patients with type 2 diabetes and nephropathy. N Engl J Med 2001;345: 861-869.

14 Lewis EJ, Hunsicker LG, Clarke WR, Berl T, Pohl MA, Lewis JB, Ritz E, Atkins RC, Rohde $\mathrm{R}, \mathrm{Raz}$ I: Renoprotective effect of the angiotensin-receptor antagonist irbesartan in patients with nephropathy due to type 2 diabetes. N Engl J Med 2001;345:851-860.

15 Kunz R, Friedrich C, Wolbers M, Mann JF: Meta-analysis: effect of monotherapy and combination therapy with inhibitors of the renin-angiotensin system on proteinuria in renal disease. Ann Intern Med 2008;148:3048.

16 Rossing P, Hommel E, Smidt UM, Parving $\mathrm{HH}$ : Reduction in albuminuria predicts a beneficial effect on diminishing the progression of human diabetic nephropathy during antihypertensive treatment. Diabetologia 1994;37:511-516.

17 Apperloo AJ, de Zeeuw D, de Jong PE: Shortterm antiproteinuric response to antihypertensive treatment predicts long-term GFR decline in patients with non-diabetic renal disease. Kidney Int Suppl 1994;45:S174S178.

18 De Zeeuw D, Remuzzi G, Parving HH, Keane WF, Zhang Z, Shahinfar S, Snapinn S, Cooper ME, Mitch WE, Brenner BM: Proteinuria, a target for renoprotection in patients with type 2 diabetic nephropathy: lessons from RENAAL. Kidney Int 2004;65:23092320.

19 Eijkelkamp WB, Zhang Z, Remuzzi G, Parving HH, Cooper ME, Keane WF, Shahinfar S, Gleim GW, Weir MR, Brenner BM, de Zeeuw $\mathrm{D}$ : Albuminuria is a target for renoprotective therapy independent from blood pressure in patients with type 2 diabetic nephropathy: post-hoc analysis from the Reduction of Endpoints in NIDDM with the Angiotensin II Antagonist Losartan (RENAAL) trial. J Am Soc Nephrol 2007;18:1540-1546.

20 Atkins RC, Briganti EM, Lewis JB, Hunsicker LG, Braden G, Champion de Crespigny PJ,
DeFerrari G, Drury P, Locatelli F, Wiegmann TB, Lewis EJ: Proteinuria reduction and progression to renal failure in patients with type 2 diabetes mellitus and overt nephropathy. Am J Kidney Dis 2005;45:281287.

21 Parving $\mathrm{HH}$, Lehnert $\mathrm{H}$, Brochner-Mortensen J, Gomis R, Andersen S, Arner P: The effect of irbesartan on the development of diabetic nephropathy in patients with type 2 diabetes. N Engl J Med 2001;345:870-878.

22 Rossing K, Christensen PK, Andersen S, Hovind $\mathrm{P}$, Hansen HP, Parving HH: Comparative effects of irbesartan on ambulatory and office blood pressure: a substudy of ambulatory blood pressure from the Irbesartan in Patients with Type 2 Diabetes and Microalbuminuria study. Diabetes Care 2003;26: 569-574.

23 Mogensen CE, Neldam S, Tikkanen I, Oren S, Viskoper R, Watts RW, Cooper ME: Randomised controlled trial of dual blockade of renin-angiotensin system in patients with hypertension, microalbuminuria, and noninsulin-dependent diabetes: the Candesartan and Lisinopril Microalbuminuria (CALM) study. BMJ 2000;321(7274):14401444.

- 24 Nakao N, Yoshimura A, Morita H, Takada $\mathrm{M}$, Kayano T, Ideura T: Combination treatment of angiotensin-II receptor blocker and angiotensin-converting-enzyme inhibitor in non-diabetic renal disease (COOPERATE): a randomised controlled trial. Lancet 2003; $361: 117-124$

25 Kunz R, Wolbers M, Glass T, Mann JF: The COOPERATE trial: a letter of concern. Lancet 2008;371:1575-1576.

- 26 Bidani A: Controversy about COOPERATE ABPM trial data. Am J Nephrol 2006;26: 629-632.

27 Retraction - Combination treatment of angiotensin-II receptor blocker and angiotensin-converting-enzyme inhibitor in nondiabetic renal disease (COOPERATE): a randomised controlled trial. Lancet 2009; 374:1226.

28 Yusuf S, Teo KK, Pogue J, Dyal L, Copland I, Schumacher H, Dagenais G, Sleight P, Anderson C: Telmisartan, ramipril, or both in patients at high risk for vascular events. $\mathrm{N}$ Engl J Med 2008;358:1547-1559. 
-29 Mann JF, Schmieder RE, McQueen M, Dyal L, Schumacher H, Pogue J, Wang X, Maggioni A, Budaj A, Chaithiraphan S, Dickstein K, Keltai M, Metsarinne K, Oto A, Parkhomenko A, Piegas LS, Svendsen TL, Teo KK, Yusuf S: Renal outcomes with telmisartan, ramipril, or both, in people at high vascular risk (the ONTARGET study): a multicentre, randomised, double-blind, controlled trial. Lancet 2008;372:547-553.

- 30 Jafar TH, Stark PC, Schmid CH, Landa M, Maschio G, Marcantoni C, de Jong PE, de Zeeuw D, Shahinfar S, Ruggenenti P, Remuzzi G, Levey AS: Proteinuria as a modifiable risk factor for the progression of nondiabetic renal disease. Kidney Int 2001;60: 1131-1140.
31 Vogt L, Navis G, de Zeeuw D: Individual titration for maximal blockade of the reninangiotensin system in proteinuric patients: a feasible strategy? J Am Soc Nephrol 2005; 16(suppl 1):S53-S57.

32 Frimodt-Moller M, Hoj Nielsen A, Strandgaard S, Kamper AL: Feasibility of combined treatment with enalapril and candesartan in advanced chronic kidney disease. Nephrol Dial Transplant 2010;25:842-847.

33 Jafar TH, Stark PC, Schmid CH, Landa M, Maschio G, de Jong PE, de Zeeuw D, Shahinfar S, Toto R, Levey AS: Progression of chronic kidney disease: the role of blood pressure control, proteinuria, and angiotensin-converting enzyme inhibition: a patientlevel meta-analysis. Ann Intern Med 2003; 139:244-252.
34 De Zeeuw D, Heerspink HL, Gansevoort RT, Bakker SJL: How to improve renal outcome in diabetes and hypertension - the importance of early screening for and treatment of microalbuminuria. Eur Nephrol 2009;3:1315.

35 Maione A, Nicolucci A, Craig JC, Tognoni G, Moschetta A, Palasciano G, Pugliese G, Procaccini DA, Gesualdo L, Pellegrini F, Strippoli GF: Protocol of the Long-term Impact of RAS Inhibition on Cardiorenal Outcomes (LIRICO) randomized trial. J Nephrol 2007;20:646-655.

\section{Editorial Comment}

Meguid El Nahas, Sheffield

This review by Heerspink and de Zeeuw is timely in view of growing concern over the use of dual blockade of the RAAS. It comes in the wake of the concern raised by the ONTARGET publication relating to potential harm of combination therapy with an ACE inhibitor and an angiotensin receptor blocker in high-risk patients. The review highlights the well-known advantages of the inhibition of RAAS in CKD and gives a comprehensive explanation as to the observed limitations of such an approach. This editor would also like to remind readers that whilst inhibition of the RAAS appeared to be one of the great achievements of the last 25 years in the management of CKD and in particular diabetic nephropathy, it is not without its limitations in terms of weak evidence of superiority compared to other antihypertensive agents in non- or even moderate- proteinuric states and non-diabetic nephropathy [Locatelli et al.: Inhibition of the renin-angiotensin system in chronic kidney disease: a crit- ical look to single and dual blockade. Nephron Clin Pract 2009;113:c286-c293]. It is also very important for nephrologists to be cautious in the elderly, the majority of patients with type 2 diabetes mellitus, who in the absence of proteinuria may have predominantly atherosclerotic hypertensive disease and ischemic nephropathy where the use of such agents or combination can do more harm than good. These are often the very patients whose heart failure prompts cardiologists to initiate RAAS. The first question I ask when I consult on an elderly patient with acute on chronic renal failure is: 'Is he on an ACE inhibitor or ARB'; the answer is often 'Yes'! Thorough explanation as in the minireview of Heerspink and de Zeeuw and ongoing education of primary care physicians and nephrologists as to the advantage but also limitations and contraindications of inhibitors of the RAAS is essential for such an approach to remain well targeted. 